Article

\title{
Cyclophilin A/EMMPRIN Axis Is Involved in Pro-Fibrotic Processes Associated with Thoracic Aortic Aneurysm of Marfan Syndrome Patients
}

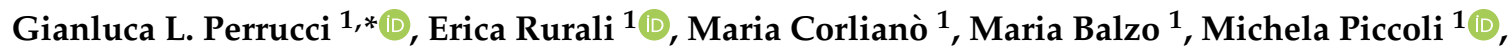 \\ Donato Moschetta ${ }^{1}(\mathbb{D})$, Alessandro Pini ${ }^{2,3}$, Raffaella Gaetano ${ }^{4}$, Carlo Antona ${ }^{5}$, Gustavo Egea ${ }^{6}(\mathbb{D}$, \\ Gunter Fischer ${ }^{7}$, Miroslav Malešević ${ }^{8}$, Francesco Alamanni ${ }^{9,10}$, Elisa Cogliati ${ }^{11}$, \\ Adolfo Paolin ${ }^{11}$, Giulio Pompilio ${ }^{1,10,12,+}$ and Patrizia Nigro ${ }^{1, \dagger}$ \\ 1 Unità di Medicina Rigenerativa e Biologia Vascolare, Centro Cardiologico Monzino-IRCCS, 20138 Milan, \\ Italy; erica.rurali@ccfm.it (E.R.); maria.corliano@gmail.com (M.C.); mariabalzo103@gmail.com (M.B.); \\ michela.piccoli93@hotmail.it (M.P.); donato.moschetta@ccfm.it (D.M.); giulio.pompilio@ccfm.it (G.P.); \\ patrizianigro@gmail.com (P.N.) \\ 2 Centro Malattie Rare, Marfan Clinic, U.O. Cardiologia, ASST FBF-Sacco, 20157 Milan, Italy; \\ alessandro.pini@asst-fbf-sacco.it \\ 3 Centro di Cardiogenetica Vascolare, IRCCS Policlinico San Donato, San Donato Milanese, 20097 Milan, Italy \\ 4 Consiglio Nazionale delle Ricerche (CNR), Istituto di Ricerca e di Innovazione Biomedica (IRIB), \\ 90146 Palermo, Italy; raffaella.gaetano@ibim.cnr.it \\ 5 Dipartimento di Scienze Biomediche e Cliniche "L. Sacco", Università degli Studi, 20157 Milan, Italy; \\ carlo.antona@unimi.it \\ 6 Departament Biomedicina, Universitat de Barcelona and Institut d'Investigacions Mèdiques August Pi i \\ Sunyer (IDIBAPS), 08036 Barcelona, Spain; gegea@ub.edu \\ 7 Max-Planck Institute for Biophysical Chemistry, 37077 Göttingen, Germany; gunter.fischer@mpibpc.mpg.de \\ 8 Enzymology department, Institute of Biochemistry and Biotechnology, Martin-Luther-University \\ Halle-Wittenberg, 06120 Halle, Germany; miroslav.malesevic@biochemtech.uni-halle.de \\ 9 Unità Operativa di Chirurgia Cardiaca, Centro Cardiologico Monzino IRCCS, 20138 Milan, Italy; \\ francesco.alamanni@unimi.it \\ 10 Dipartimento di Scienze Cliniche e di Comunità, Università degli Studi, 20122 Milan, Italy \\ 11 Treviso Tissue Bank Foundation, 31100 Treviso, Italy; ecogliati@fbtv-treviso.org (E.C.); \\ adolfo.paolin54@gmail.com (A.P.) \\ 12 Dipartimento di Chirurgia Cardiovascolare, Centro Cardiologico Monzino IRCCS, 20138 Milan, Italy \\ * Correspondence: gianluca.perrucci@ccfm.it; Tel.: +39-02-5800-2754; Fax: +39-02-5800-2342 \\ + Equal contribution as last authors.
}

Received: 4 November 2019; Accepted: 3 January 2020; Published: 8 January 2020

check for updates

\begin{abstract}
Background: Marfan syndrome (MFS) is a genetic disease, characterized by thoracic aortic aneurysm (TAA), which treatment is to date purely surgical. Understanding of novel molecular targets is mandatory to unveil effective pharmacological approaches. Cyclophilin A (CyPA) and its receptor EMMPRIN are associated with several cardiovascular diseases, including abdominal aortic aneurysm. Here, we envisioned the contribution of CyPA/EMMPRIN axis in MFS-related TAA. Methods: We obtained thoracic aortic samples from healthy controls (HC) and MFS patients' aortas and then isolated vascular smooth muscle cells (VSMC) from the aortic wall. Results: our findings revealed that MFS aortic tissue samples isolated from the dilated zone of aorta showed higher expression levels of EMMPRIN vs. MFS non-dilated aorta and HC. Interestingly, angiotensin II significantly stimulated CyPA secretion in MFS-derived VSMC (MFS-VSMC). CyPA treatment on MFS-VSMC led to increased levels of EMMPRIN and other MFS-associated pro-fibrotic mediators, such as TGF- $\beta 1$ and collagen I. These molecules were downregulated by in vitro treatment with CyPA inhibitor MM284. Our results suggest that CyPA/EMMPRIN axis is involved in MFS-related TAA development, since EMMPRIN is upregulated in the dilated zone of MFS patients' TAA and the
\end{abstract}


inhibition of its ligand, CyPA, downregulated EMMPRIN and MFS-related markers in MFS-VSMC. Conclusions: these insights suggest both a novel detrimental role for CyPA/EMMPRIN axis and its inhibition as a potential therapeutic strategy for MFS-related TAA treatment.

Keywords: thoracic aortic aneurysm; EMMPRIN; fibrosis; vascular smooth muscle cells; Cyclophilin A

\section{Introduction}

Marfan syndrome (MFS) is a connective tissue disease with an autosomal dominant inheritance and an estimated incidence of 1:5000 individuals [1]. This disorder is caused by mutations in the FBN1 gene encoding fibrillin-1, an extracellular matrix (ECM) protein that provides elasticity and structural support to several tissues [2]. At the molecular level, fibrillin-1 forms complex extracellular structures, called microfibrils, which modulate elastic fiber biogenesis and homeostasis and regulate the bioavailability/activity of different growth factors [3].

FBN1 mutations lead to impaired fibrillin-1 protein synthesis, secretion and/or incorporation in ECM, determining a microfibrillar architecture degeneration and destruction of the ECM integrity [4]. As a consequence, this genetic defect leads to pleiotropic manifestations, such as skeletal overgrowth, ocular lens luxation (i.e., ectopia lentis), and cardiovascular events as the progressive aortic-root dilation [5]. Overall, cardiovascular impairments dominate MFS patients' prognosis. Indeed, the thoracic aortic dilatations worsening culminate in thoracic aortic aneurysms (TAA), which, in turn, often lead to the major cause of death in MFS patients as aortic dissection [1].

It is noteworthy that the therapy for MFS-associated TAA is, to date, purely surgical. Unfortunately, an effective pharmacological treatment for this life-threatening disease in MFS patients still remains unknown. Thus, the complete identification of the mechanisms contributing to MFS aneurysm formation and the subsequent identification of novel therapeutic targets are clinical imperatives for this patients' category [6].

Over the past 25 years, novel insights were gained in the molecular biology of MFS, which have implicated the transforming growth factor- $\beta$ (TGF- $\beta$ )- and angiotensin II (AngII)-dependent pathways $[7,8]$ as key culprits in TAA development. These findings suggest that other factors, in association with FBN1 mutations, may play an important role in TAA development and progression. However, a complete picture of the pathogenic mechanisms driving the onset and progression of aortic disease in MFS has not been achieved.

The receptor named extracellular matrix metalloproteinase (MMP) inducer (EMMPRIN) is strongly associated with several cardiovascular diseases [9]. Interestingly, EMMPRIN acts not only as an MMP activator, but also as a classical receptor regulating downstream pathways, specifically those involved in fibrosis and inflammation [10]. Of note, it was previously reported that EMMPRIN levels are strongly upregulated in VSMC of human non-syndromic TAA and its expression is induced in vitro by TGF- $\beta$ and AngII [11-13]. Among EMMPRIN ligands important to be mentioned there are E-selectin, S100A9, and Cyclophilin A (CyPA) [14]. The latter is a peptidyl-prolyl cis-trans isomerase, object of several studies in the cardiovascular field $[15,16]$. Notably, we demonstrated that the deletion of CyPA in mice prevents the formation of abdominal aortic aneurysm (AAA) in response to AngII infusion [17]. Remarkably, we also showed that the expression of CyPA in VSMC, rather than in bone marrow-derived cells, is crucial in AAA development. To establish CyPA relevance in human aneurysmal disease, we discovered that AngII causes its release from VSMC derived from human AAA together with the activation of MMP-2. As EMMPRIN ligand, it was reported that CyPA/EMMPRIN binding activates ERK1/2 signaling pathway [18] and promotes also MMP cleavage. However, the contribution of CyPA/EMMPRIN axis in MFS-associated TAA has not been elucidated yet.

Here, we aimed to fill this gap, providing evidence about the role of CyPA/EMMPRIN axis as the molecular player in MFS and as a novel attractive therapeutic target. Specifically, we found that 
CyPA (i) is secreted from VSMC obtained by MFS patients' TAA samples after treatment with AngII and (ii) orchestrates the activation of deleterious pathways leading to fibrosis in MFS through the upregulation and activation of EMMPRIN.

\section{Materials and Methods}

\subsection{Data Availability}

The data that support the findings of this study are available from the corresponding author upon reasonable request.

\subsection{Patients Enrollment}

Aortic tissue samples were collected in the surgery room from the dilated and non-dilated zone of MFS patients' aortic aneurysm. From the same cohort of patients, and plasma samples were collected before the surgery procedure. The healthy control (HC) samples were provided by Treviso Tissue Bank Foundation. All MFS patients gave written informed consent for tissue collection and the procedure was approved by ASST FBF-Sacco Ethics Committee (Prot. N 39138/2016, Milan, Italy). Aortic samples were stored in serum-free Medium 231 (Life Technologies, Carlsbad, CA, USA) and then divided into fragments dedicated to histology and molecular studies.

\subsection{Immunostaining and Histological Assays}

Aortic fragments assigned to histological studies were fixed in Carnoy's solution, paraffin-embedded and 5 - $\mu \mathrm{m}$-thick sections were cut from each sample. To evaluate vascular elastic fiber integrity and collagen content in tissue, sections were stained with the Verhoeff-Van Gieson solution (Bio-Optica, Milan, Italy) following the manufacturer's protocol. To perform immunohistological studies, sections were incubated with primary antibodies against CyPA (R\&D system, Minneapolis, MN, USA) and EMMPRIN, also called CD147 (R\&D System, Minneapolis, MN, USA), over-night $(\mathrm{O} / \mathrm{N})$ at $4{ }^{\circ} \mathrm{C}$. As a negative control, species- and isotype-matched IgGs were incubated in place of primary antibodies. Slides were viewed with AxioScope microscope equipped with AxioCam camera (Carl Zeiss, Oberkochen, Germany). Densitometric analyses and elastic fiber length measurements were performed with AxioVision 4.7 software (Carl Zeiss, Oberkochen, Germany) by two blinded readers.

\subsection{Aortic VSMC Isolation}

Samples from aortic tunica media, stored in serum-free Medium 231 (Life Technologies, Carlsbad, CA, USA), were washed with Phosphate Buffer Saline (PBS), minced and digested $\mathrm{O} / \mathrm{N}$ at $37^{\circ} \mathrm{C}$ in a solution of $2 \mathrm{mg} / \mathrm{mL}$ collagenase type II (Worthington Biochemical Corporation, Lakewood, NJ, USA) in complete Medium 231, supplemented with Smooth Muscle Growth Supplement (SMGS, Life Technologies, Carlsbad, CA, USA). Then, the result of tissue digestion was filtered with $100 \mu \mathrm{m}$ cell strainer, pelleted and plated in complete Medium 231. To improve cell growth, the day after isolation the medium was changed to remove the residual erythrocytes. Regarding MFS samples, cells used for the in vitro experiments were those isolated only by non-dilated zone.

\subsection{ELISA Assays}

For each patient enrolled in the study, $8 \mathrm{~mL}$ of peripheral whole blood was collected using EDTA Vacutainer tubes (Becton Dickinson, Franklin Lakes, NJ, USA). Plasma was obtained centrifuging the whole blood for $10 \mathrm{~min}$ at $2500 \times g$, at $4{ }^{\circ} \mathrm{C}$. CyPA serum levels of HC subjects and MFS patients were detected with an ELISA kit (USCN Life Science Inc., Wuham, China) following the manufacturer's instructions.

TGF- $\beta 1$ levels in VSMC conditioned medium after five days of $100 \mathrm{ng} / \mathrm{mL}$ CyPA and/or $250 \mathrm{ng} / \mathrm{mL}$ MM284 (CyPA inhibitor) treatment were detected with a sandwich Quantikine ${ }^{\circledR}$ ELISA kit (R\&D System, Minneapolis, MN, USA) following the manufacturer's instructions. 


\subsection{MRNA Extraction and qRT-PCR Assay}

After collection, aortic samples from healthy controls (HC) and Marfan syndrome patients (MFS) were stored at $-80^{\circ} \mathrm{C}$ in an appropriate amount of RNA Later solution (Sigma-Aldrich, Saint Louis, MO, USA). Once defrosted, aortic dried tissues were crushed by the Bessman Tissue Pulverizer (Spectrum Europe BV, Breda, Netherlands) and RNA was isolated with the TRIzol ${ }^{\circledR}$ solution (ThermoFisher Scientific, Paislay, Scotland, UK). In parallel, after cell isolation, HC- and MFS-VSMC were treated with $100 \mathrm{ng} / \mathrm{mL}$ CyPA, $5 \mathrm{ng} / \mathrm{mL}$ TGF- $\beta 1,1 \mu \mathrm{M}$ AngII, $250 \mathrm{ng} / \mathrm{mL}$ MM284, $5 \mu \mathrm{g} / \mathrm{mL}$ EMMPRIN blocking antibody (Ancell, Bayport, MN, USA), or $5 \mu \mathrm{g} / \mathrm{mL} \mathrm{IgG}$ isotype antibody (Ancell, Bayport, MN, USA). Cellular RNA was isolated by using a Total RNA Purification kit (Norgen Biotek corp., Thorold, Canada). Both for tissues and cells, the quantification of isolated RNA was determined with a spectrophotometer (ND-1000, NanoDrop ${ }^{\circledR}$, EuroClone $^{\circledR}$ ). Reverse transcription was conducted with the SuperScript III (Invitrogen, Carlsbad, CA, USA) following the manufacturer's instructions. qRT-PCR was performed with the use of the iQTM SYBR Green Super Mix (Bio-Rad Laboratories, Hercules, CA, USA). All reactions were performed in a 96-well format with the iQ5TM (Bio-Rad Laboratories, Hercules, CA, USA). The relative quantities of specific mRNA were obtained with the use of the comparative $\mathrm{Ct}$ method and were normalized to the Ribosomal Protein L32 (RPL32).

\subsection{Western Blot and Slot Blot Analyses}

Both aortic tissues and VSMC were lysed in cell lysis buffer (Cell Signaling Technology, Danvers, MA, USA) supplemented with protease and phosphatase inhibitor cocktails (Sigma-Aldrich, Saint Louis, MO, USA). Total protein extracts were subjected to SDS-PAGE and transferred onto a nitrocellulose membrane. The membranes were blocked for $1 \mathrm{~h}$ at room temperature in $5 \%$ non-fat dry milk in Wash Buffer (Tris Buffer Sulfate $1 \times, 0.1 \%$ Tween 20) and then incubated $\mathrm{O} / \mathrm{N}$ at $4{ }^{\circ} \mathrm{C}$ with the appropriate primary antibody. For Slot blot analysis, $100 \mu \mathrm{L}$ of VSMC conditioned media were directly loaded on nitrocellulose membrane with a pore size of $0.2 \mu \mathrm{m}$ (Bio-Rad Laboratories, Hercules, CA, USA) by vacuum filtration. The membranes were blocked for $1 \mathrm{~h}$ at room temperature in $5 \%$ non-fat dry milk (Blotto, ChemCruz, Huissen, Netherlands) in Wash Buffer (Tris Buffer Sulfate 1×, 0.1\% Tween 20) and then incubated $\mathrm{O} / \mathrm{N}$ at $4{ }^{\circ} \mathrm{C}$ with the appropriate primary antibody. Primary antibodies used were specific for phospho-SMAD2/3 (Cell Signaling Technology, Danvers, MA, USA), SMAD2/3 (Cell Signaling Technology, Danvers, MA, USA), phospho-ERK1/2 (Cell Signaling Technology, Danvers, MA, USA), ERK1/2 (Cell Signaling Technology, Danvers, MA, USA), CyPA (R\&D System, Minneapolis, MN, USA), EMMPRIN (R\&D System, Minneapolis, MN, USA), AngII type 1 receptor (AT1R, AbCam, Cambridge, UK), and GAPDH (Santa Cruz Biotechnology, Dallas, TX, USA). The membranes were incubated with peroxidase-conjugated secondary antibodies (GE Healthcare, Chicago, IL, USA) for $1 \mathrm{~h}$. Signals were visualized using the enhanced chemiluminescence Western blotting detection system (ThermoFisher Scientific, Paislay, Scotland, UK). Images were acquired with the Alliance Mini 2M (UVITec, Cambridge, UK), and densitometric analysis of membranes was performed using the Alliance Mini 416.07 software (UVITec, Cambridge, UK). Proteins were normalized according to the GAPDH signal.

\subsection{ImageStream $X$ Imaging Flow Cytometry}

For CyPA and EMMPRIN cellular localization experiments, VSMC were acquired by the ImageStreamX imaging cytometer (Amnis Corporation, Seattle, WA, USA), with 40× magnification and low flow rate/high sensitivity using the INSPIRE software. VSMC were treated with $100 \mathrm{ng} / \mathrm{mL}$ CyPA for $3 \mathrm{~h}$, and then gently detached from Petri dishes by using the TrypLE ${ }^{\text {тм }}$ Select solution (ThermoFisher Scientific, Paislay, Scotland, UK). Afterward, VSMC were pelleted, resuspended in FACS buffer ( $1 \times$ PBS, $0.1 \%$ Bovine Serum Albumin (BSA), and $5 \mathrm{mM}$ EDTA) and incubated with conjugated primary antibodies for $10 \mathrm{~min}$ at room temperature. In the case of unconjugated primary antibody, cells were washed, resuspended in FACS buffer and incubated with a specific secondary 
antibody conjugated with a fluorescent dye. Data from 10,000 events per sample were collected and the percentage of the elements was calculated using the IDEA software (Amnis Corporation, Seattle, WA, USA). To assess the nuclear translocation of CyPA, primary antibody against CyPA (R\&D system), secondary antibody FITC-conjuagated, and DRAQ5 nuclear dye (AbCam, Cambridge, UK) were used. In order to evaluate the membrane localization of EMMPRIN, a primary antibody against EMMPRIN-APC (TRA-1-85 Allophycocyanin MAb, Clone TRA-1-85, R\&D System, Minneapolis, MN, USA) was used. As cell membrane control, primary antibody against human leukocyte antigen-ABC (HLA-ABC) conjugated with phycoerythrin (PE) fluorescent dye (BD Pharmigen, San Jose, CA, USA) was used.

\subsection{Sircol Assay}

Total soluble collagen content in cell lysates and supernatant from HC- and MFS-VSMC, treated for five days with $100 \mathrm{ng} / \mathrm{mL}$ CyPA and/or $250 \mathrm{ng} / \mathrm{mL}$ MM284, was measured using Sircol soluble collagen assays (Biocolor, Carrickfergus, UK) as described in the manufacturer's protocol. The quantity of collagen was calculated according to the kit standard curve.

\subsection{Statistical Analyses}

Quantitative results are expressed as mean \pm SD. Statistical significance was evaluated with GraphPad Prism 5. Variables were analyzed by two-way ANOVA with Bonferroni's post-test or Student's $t$-test, as appropriate. A value of $p \leq 0.05$ was deemed statistically significant.

\section{Results}

\subsection{MFS Patients' Thoracic Aortic Aneurysm Shows Increased Fibrosis and Activation of TGF- $\beta 1$ Signaling}

To characterize TAA in MFS, we collected bioptic samples from dilated and non-dilated aorta of patients undergoing aortic replacement. MFS specimens were compared with thoracic aortic samples of HC. We evaluated the expression of principal genes involved in MFS disease by using total RNA extracts from the ascending aortic tissues. qRT-PCR analyses showed an upregulation of genes encoding several pro-fibrotic factors, such as collagen I (COL1A1) and connective tissue growth factor (CTGF) in aortic MFS patients' samples vs. HC (Figure 1a). Similar results were obtained for SMTN, a gene encoding the typical VSMC marker smoothelin, and genes related to TGF- $\beta 1$, such as TGFB1 itself, type 1 TGF- $\beta$ receptor (TGFBR1), and latent TGF- $\beta$ binding protein 1 (LTBP1).

To further investigate on the aortic wall structural integrity, we examined the elastic fiber disorganization/fragmentation and the collagen deposition, both typical features of MFS aortic tissue, by using the Verhoeff-Van Gieson staining. This specific staining highlights the former feature in black and the latter in pink/red (Figure 1b). As expected, we found an evident elastin fragmentation, evaluated and summarized in Table 1 as elastic fiber length, and a higher amount of collagen deposition in MFS samples when compared with HC (Figure 1c).

In order to assess the well-known over-activation of TGF- $\beta 1$ pathways in MFS, we performed a Western blot analysis on total protein extracts of thoracic aortic samples isolated from HC and MFS patients (Figure 1d). As expected, we found a significant activation, in terms of higher expression levels of phosphorylated forms, of both transcription factors SMAD2/3 (Figure 1e) and ERK1/2 (Figure 1f) in MFS samples versus $\mathrm{HC}$.

Altogether, these data confirm the pathologic events in patients' samples and also support the key role of pro-fibrotic factors in the pathogenesis of MFS-associated TAA. 


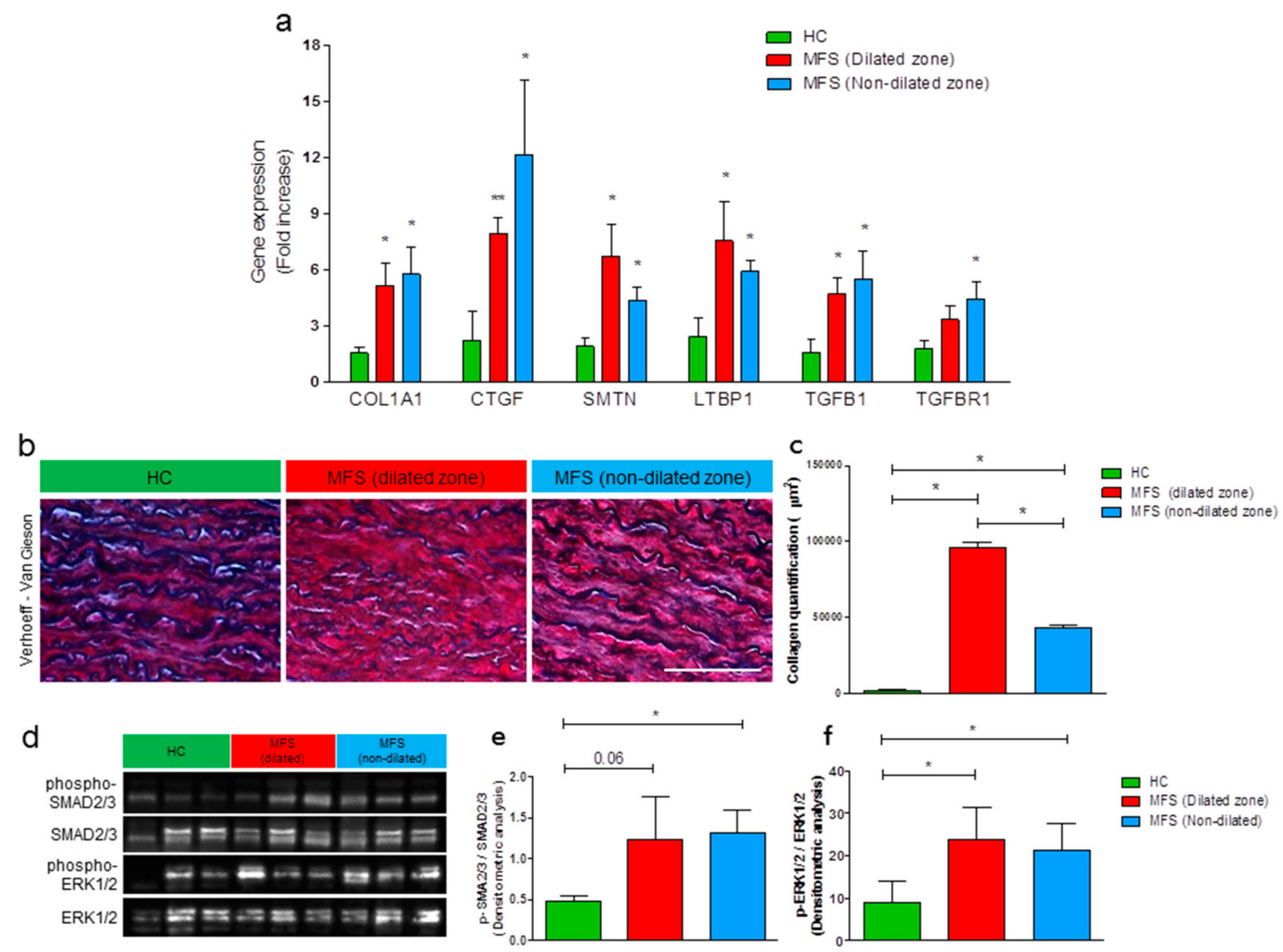

Figure 1. Collagen deposition, disease-related gene expression, and TGF- $\beta$-dependent pathways activation are higher in thoracic aortas of Marfan syndrome (MFS) patients than healthy controls. (a) Expression of MFS-related genes in total RNA extracts of thoracic aortas from healthy controls (HC) (green bars), MFS dilated (red bars), and MFS non-dilated zones (blue bars). qRT-PCR analyses have been performed in triplicate and data are shown as fold change $\pm \mathrm{SD}, \mathrm{n}=5$. Student's $t$-test: ${ }^{*} p<0.05$, ** $p<0.01$. (b) Representative images of Verhoeff-Van Gieson staining on HC (left panel) and MFS patient aortas (dilated zone, central panel; non-dilated zone, right panel). Magnification $=20 \times$. Scale bar $=200 \mu \mathrm{m}$. (c) Collagen quantification data are shown as mean $\pm \mathrm{SD}, \mathrm{n}=5$. Student's $t$-test: ${ }^{*} p<0.05$. (d) Western Blot of active phosphorylated form and total SMAD2/3 and ERK1/2 in total protein extracts of thoracic aortas from HC (green bars), MFS dilated (red bars), and MFS non-dilated zones (blue bars), and relative quantification. Data are shown as mean $\pm \mathrm{SD}, \mathrm{n}=5$. Student's $t$-test: ${ }^{*} p<0.05$. (e,f) Quantification of phospho-SMAD2/3 (e) and phospho-ERK1/2 (f) Western blot on thoracic aortic tissues. Data are shown as mean $\pm \mathrm{SD}, \mathrm{n}=5$. Student's $t$-test: ${ }^{*} p<0.05$.

Table 1. Elastic fiber length measurement in thoracic aortas of healthy controls (HC) and MFS patients.

\begin{tabular}{ccc}
\hline & Elastic Fiber Length Mean $(\mu \mathrm{m} \pm \mathrm{SD})$ & $p$ \\
\hline HC & $235.94 \pm 64.78$ & $/$ \\
MFS (dilated zone) & $116.07 \pm 54.64$ & $*$ \\
MFS (non-dilated zone) & $97.93 \pm 37.68$ & $*$ \\
\hline healthy controls; MFS = Marfan syndrome. ${ }^{*}=$ Student's $t$-test $p$-value $<0.05$ (vs. HC).
\end{tabular}

\subsection{Expression Levels of EMMPRIN Are Higher in MFS vs. HC, both in vivo and in vitro}

Based on a strong association between pathways/functions regulated by CyPA/EMMPRIN axis and major occurring events in MFS, we started our analysis of EMMPRIN and CyPA expression levels in MFS tissues compared to HC. Data described in Figure 2 provided evidence for an upregulation of EMMPRIN gene (Figure 2a) and protein (Figure 2c-e), specifically in the dilated zone of MFS aortic tissues when compared with HC samples and non-dilated zone of MFS aorta, both by Western blot 
(Figure 2c) and immunohistochemistry (Figure 2d,e) assays. Additionally, in an attempt to investigate the activator of this axis, we measured CyPA expression. Results revealed that CyPA expression is not modulated in MFS vs. HC aortic specimens, by qRT-PCR (Figure 2b), by Western blot (Figure 2c), and by immunohistochemistry (Figure 2f,g) analyses.

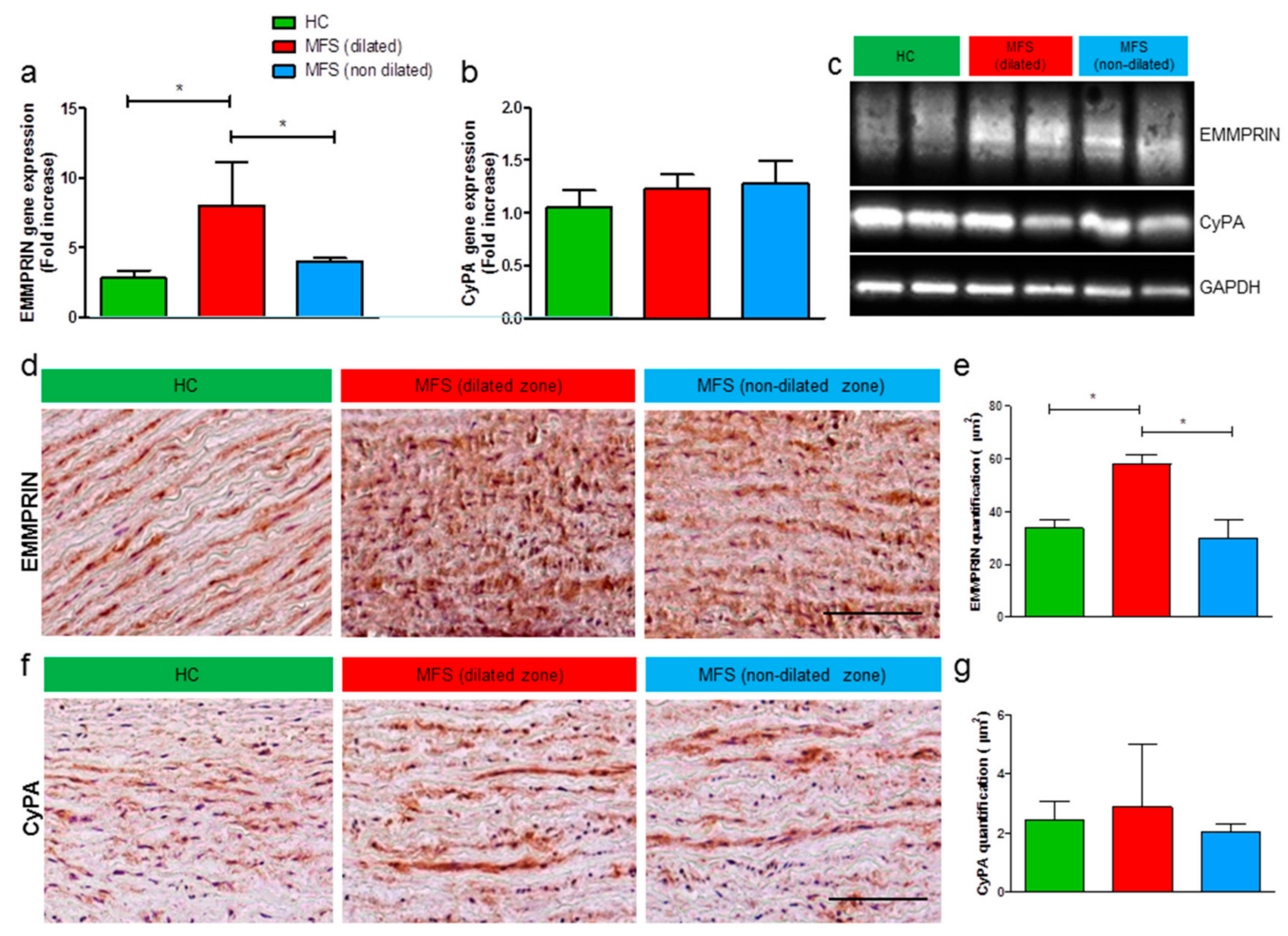

Figure 2. EMMPRIN expression levels are higher in the thoracic aorta of MFS patients. (a,b) EMMPRIN (a) and CyPA (b) gene expression in total RNA extracts of thoracic aortas from HC (green bars), MFS dilated (red bars) and MFS non-dilated zones (blue bars). qRT-PCR analyses were performed in triplicate and data are shown as fold change $\pm \mathrm{SD}, \mathrm{n}=5$. Student's $t$-test: ${ }^{*} p<0.05$. (c) Representative images of Western Blot analysis on EMMPRIN and CyPA in total protein extracts of thoracic aortas from HC (green bars), MFS dilated (red bars), and MFS non-dilated zones (blue bars). (d-g) Representative images of immunohistochemistry for EMMPRIN (d) and CyPA (f) on thoracic aorta of HC subjects (left panel) and MFS patients (dilated zone, central panel; non-dilated zone, right panel). Magnification $=20 \times$. Scale bar $=200 \mu \mathrm{m}$. Quantification of immunohistochemistry for EMMPRIN (e) and CyPA (g) has been shown as mean $\pm \mathrm{SD}, \mathrm{n}=5$. Student's $t$-test: ${ }^{*} p<0.05$.

All these data suggest an important modulation of EMMPRIN but not of endogenous CyPA in MFS aneurysmal tissue, with particular relevance for the dilated zone of MFS-associated TAA.

Since VSMC have been described as pivotal players in aneurysm pathogenesis, these cells were explanted from the non-dilated zone of ascending aorta of MFS patients specimens (MFS-VSMC) and HC (HC-VSMC) and then characterized. Supplementary Figure S1 shows immunofluorescence characterization on HC- and MFS-VSMC, in which the VSMC identity was confirmed by the expression of typical smooth muscle cell markers, such as $\alpha$-SMA and calponin. Moreover, in order to evaluate the expression of CyPA and EMMPRIN, multiple immunostainings were performed on VSMC. Specifically, EMMPRIN signal was detected together with $\alpha$-SMA (Supplementary Figure S1a), while CyPA was evaluated together with calponin (Supplementary Figure S1b). Interestingly, this analysis showed a clearly visible difference in cell areas, greater in MFS-VSMC in comparison with HC-VSMC. 
Then, qRT-PCR and Western blot analyses on VSMC ascribed the responsibility for EMMPRIN overexpression to these cells (Figure 3a-c). Similarly to tissue results, no statistical differences in CyPA expression levels (Figure 3c-e) were detected between HC- and MFS-VSMC.
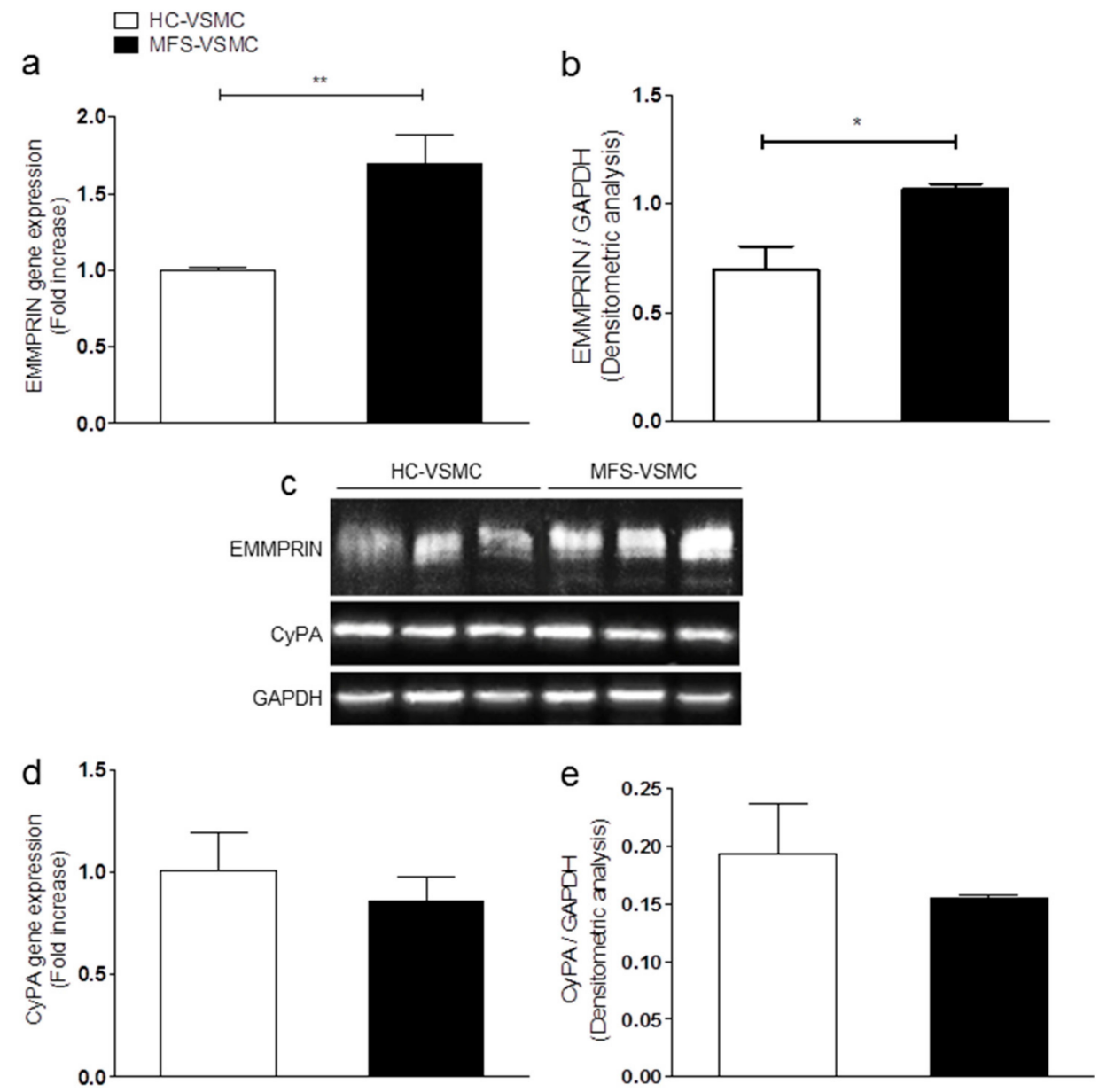

Figure 3. EMMPRIN expression levels are higher in vascular smooth muscle cells (VSMC) isolated from the thoracic aorta of MFS patients. (a,d) EMMPRIN (a) and CyPA (d) gene expression in RNA extracts of HC-VSMC (white bars) and MFS-VSMC (black bars). qRT-PCR analyses have been performed in triplicate and data are shown as fold change $\pm \mathrm{SD}, \mathrm{n}=5$. Student's $t$-test: ${ }^{*} p<0.05,{ }^{* *} p<0.01$. (c) Western Blot of EMMPRIN and CyPA proteins in total extracts of HC-VSMC and MFS-VSMC, $\mathrm{n}=3$. $(\mathbf{b}, \mathbf{e})$ Quantification of EMMPRIN (B) and CyPA (e) Western blot. Data are shown as mean $\pm \mathrm{SD}, \mathrm{n}=3$. Student's $t$-test: ${ }^{*} p<0.05$.

3.3. MFS-VSMC Show A Higher Expression of AT1R, an Enhanced Secretion of CyPA, and MFS Patients Display Higher Plasmatic CyPA Levels

To date, it is well known that CyPA can localize into the nucleus, but also in the cytoplasmic compartment. Thus, we compared CyPA specific localization in HC- and MFS-VSMC by Amnis ImageStreamX. This approach revealed that CyPA localization is prevalently cytoplasmatic, with no statistical differences between HC- and MFS-VSMC (Figure S2).

Although the initial evidence on the intracellular function of CyPA, recent studies revealed that this molecule can be secreted by several cell types in response to pathological stimuli $[17,19,20]$. In order to evaluate in our in vitro model whether CyPA is secreted after stimulation with specific MFS molecular mediators, we treated HC- and MFS-VSMC with TGF- $\beta 1$ and AngII. The former is a well-known key player of TAA formation in MFS patients, the latter is an effective chemical stimulus involved in CyPA secretion by VSMC. Importantly, in 2013 it was reported that the AngII receptor type 
1 (AT1R) is significantly upregulated in both aortic tissue and VSCM of MFS patients [21]. In order to provide a further quantitative result on AT1R protein expression levels, we performed a Western blot analysis for this AngII receptor on both aortic tissue and VSMC samples. As showed in Figure 4a,b, AT1R expression levels resulted higher both in MFS-derived samples of aortic tissue and VSMC. After that, our analysis by Slot blot assay on the VSCM conditioned medium showed, consistently with literature data, a massive increase of CyPA secretion, specifically in MFS-VSMC after AngII treatments (Figure 4c).

Several studies reported high circulating levels of CyPA in patients with numerous cardiovascular diseases [10,22-25]. Therefore, we next investigated whether circulating exogenous CyPA (CyPA) levels in MFS patients' plasma may differ from those in HC, recapitulating the in vitro results. As reported in Figure 4d, MFS patients ( $\mathrm{n}=24)$ with thoracic aortic aneurysm showed a statistically significant increase in secreted levels of CyPA when compared with age- and sex-matched HC.

These data suggest a specific alteration of CyPA/EMMPRIN axis in MFS-VSMC. In fact, while CyPA is prevalently secreted into the bloodstream, its receptor EMMPRIN is locally enhanced in TAA of MFS patients.
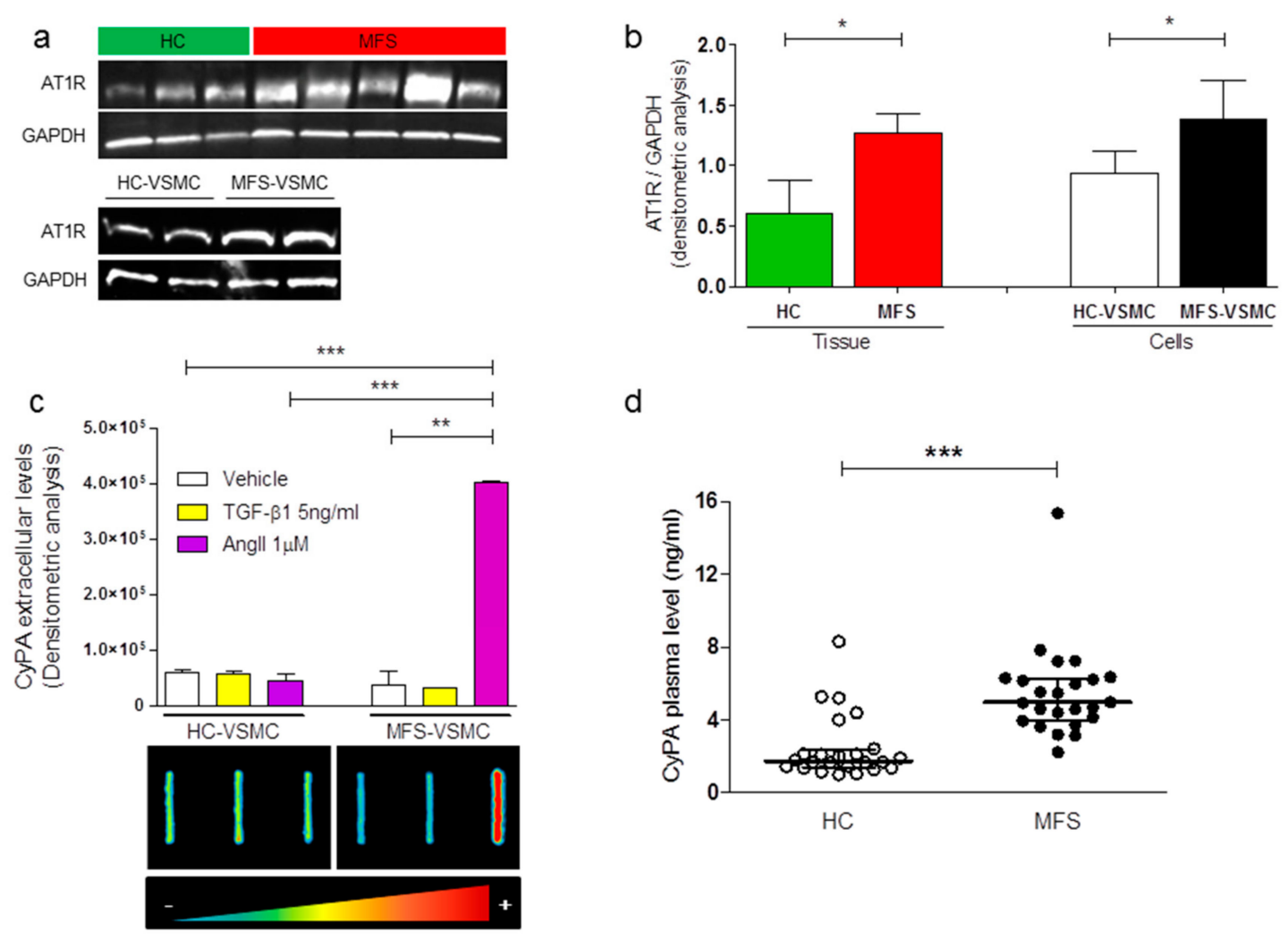

Figure 4. CyPA is secreted by MFS-VSMC after AngII treatment. (a) Western Blot of AT1R proteins in total protein extracts of thoracic aortas from HC (green bars, upper panel) and MFS dilated (red bars, upper panel), $\mathrm{n}=8$, and in total extracts of HC-VSMC and MFS-VSMC (lower panel), $\mathrm{n}=4$ for VSMC. (b) Quantification of AT1R Western blots. Data are shown as mean \pm SD. Student's $t$-test: ${ }^{*} p<0.05$. (c) Slot-blot analysis and relative quantification of CyPA secreted in the conditioned media of HCand MFS-VSMC, treated with vehicle (white bars), $5 \mathrm{ng} / \mathrm{mL}$ of TGF- $\beta 1$ (yellow bars), and $1 \mu \mathrm{M}$ AngII (purple bars), $\mathrm{n}=5$. Student's $t$-test: ${ }^{*} p<0.05,{ }^{* *} p<0.01,{ }^{* *} p<0.0001$. (d) ELISA assay on CyPA plasma levels of HC donors and MFS patients. Data are shown as median and IQR (InterQuartile Range $25-75 \%), \mathrm{n}=24$ /group. Mann-Whitney test: ${ }^{* * *} p<0.0001$. 


\subsection{CyPA Stimulates EMMPRIN Expression and Activation}

The most known EMMPRIN molecular function is that of matrix metalloprotease activator, among which the most important and active at the aneurysmal thoracic aortic tissue is MMP-2 [11-13]. In order to verify the MMP-2expression levels in the plasma of our cohort of patients as well as in the VSMC in vitro model, we performed an ELISA and a qRT-PCR assays, respectively. The results of both these assays confirmed that MMP-2 was significantly upregulated both in the plasma of MFS patients (Figure 5a) as well as gene expression in MFS-VSMC (Figure 5b).

Taking into account that CyPA is the ligand of EMMPRIN, that this binding is able in activating EMMPRIN, and that CyPA levels were higher in MFS patients' plasma as well as in conditioned medium of MFS-VSMC after AngII stimulation, we next evaluated the direct effect of the exogeneous CyPA administration on MFS-VSMC. In order to assess whether CyPA treatment may influence cell proliferation or induce MFS-VSMC apoptosis, we performed two different FACS analyses. By using BrdU, we obtained no statistical differences in cycle phases of MFS-VSMC (Figure S3a), thus CyPA does not induce cell cycle transition in MFS-VSMC. Similarly, by using the Annexin V antibody, we demonstrated that CyPA does not induce apoptosis in MFS-VSMC (Figure S3b,c).

Results showed that CyPA $(100 \mathrm{ng} / \mathrm{mL})$ treatment led to a significant overexpression of EMMPRIN gene (Figure 5c) in MFS- vs. HC-VSMC. Concerning EMMPRIN protein levels, the ImageStreamX analysis confirmed the EMMPRIN localization on VSMC membranes and further highlighted the EMMPRIN overexpression after CyPA treatment, specifically on cell membranes of MFS-VSMC (Figure 5d).
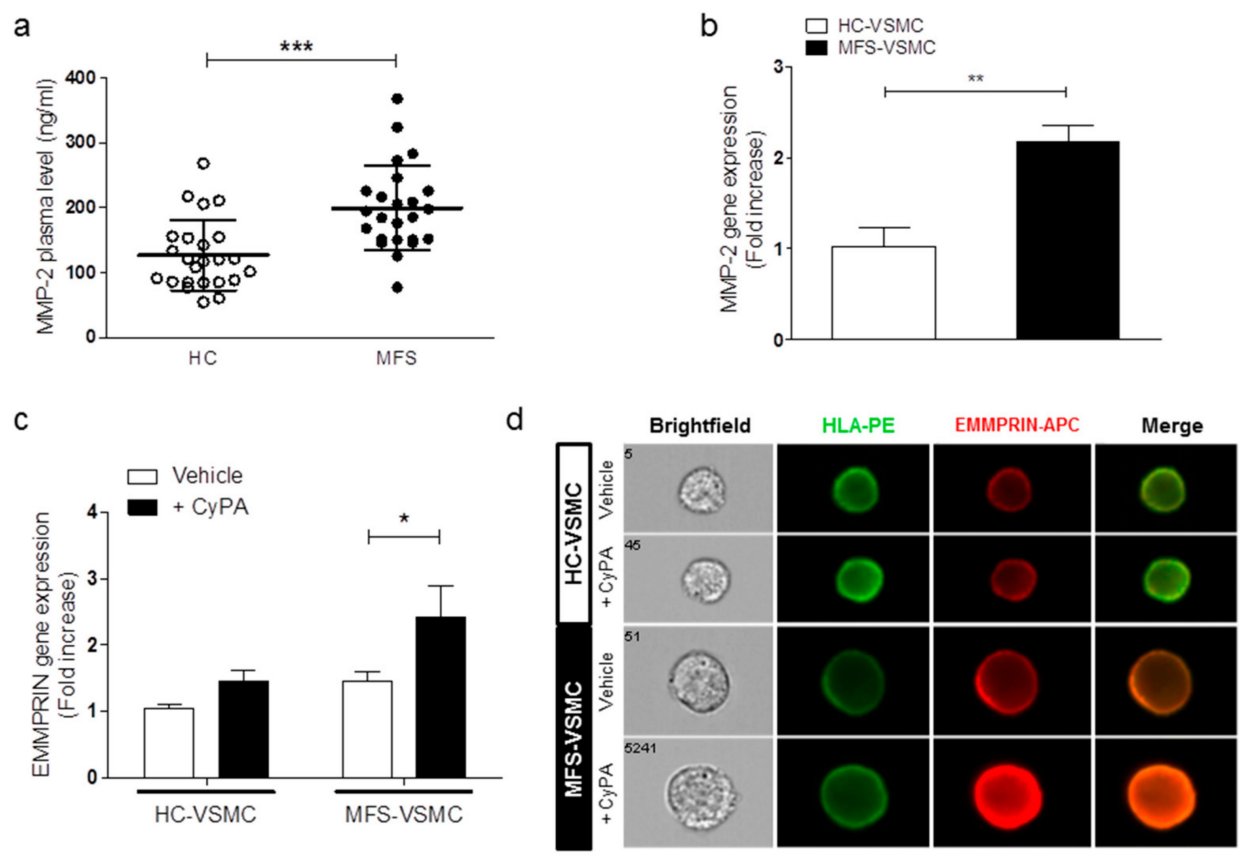

Figure 5. MMP-2 is up-regulated in MFS and exogenous CyPA promotes EMMPRIN gene and protein expression in MFS-VSMC. (a) ELISA assay on MMP-2 plasma levels of HC donors and MFS patients. Data are shown as mean $\pm \mathrm{SD}, \mathrm{n}=24$ /group. Student's $t$-test: ${ }^{* *} p<0.0001$. (b) MMP-2 gene expression in RNA extracts of HC-VSMC (white bars) and MFS-VSMC (black bars). qRT-PCR analyses have been performed in triplicate and data are shown as fold change $\pm \mathrm{SD}, \mathrm{n}=5$. Student's $t$-test: ${ }^{* *} p<0.01$. (c) EMMPRIN gene expression levels in HC-VSMC and MFS-VSMC, after $100 \mathrm{ng} / \mathrm{mL}$ CyPA treatment. qRT-PCR analyses have been performed three times in triplicate and data are shown as fold change \pm $\mathrm{SD}, \mathrm{n}=5 .{ }^{*} p<0.05$ (d) Representative images of immunofluorescence on HC-VSMC and MFS-VSMC, after $100 \mathrm{ng} / \mathrm{mL}$ CyPA treatment, in brightfield, HLA-PE antibody, EMMPRIN-APC antibody, and merge. Cell images have been generated in real-time by Amnis imaging flow cytometer ImageStreamX with a magnification of $40 \times$. 
To better understand whether EMMPRIN was functionally active in our setting, we took advantage of its capability to directly activate MMPs. Thus, HC- and MFS-VSMC were analyzed by an in situ zymography (Supplementary Methods) after treatments with CyPA and a selective antibody against the EMMPRIN extracellular domain. The immunofluorescence images showed a higher MMP activation after CyPA treatment, which is extrapolated by enhanced emission of a green-fluorescent signal by cleavages of engineered gelatin (Figure S4a). The quantification of FITC-positive areas revealed an overall higher activity of EMMPRIN in MFS- vs. HC-VSMC, and overall in MFS-VSMC after CyPA treatment (Figure S4b). Similarly, the CyPA/EMMPRIN axis inhibition mediated by MM284 led to significantly decreased levels of MMP-2 gene expression in MFS-VSMC (Figure S4c).

These data reveal that the treatment of MFS-VSMC with CyPA leads not only to a higher expression of EMMPRIN, but also to its increased activity.

\subsection{Blocking CyPA/EMMPRIN Axis Reverts the MFS-Related Expression of Pro-Fibrotic Mediators}

It is nowadays well established that MFS condition is strongly associated with the activation of TGF- $\beta$ canonical (SMAD2/3) and non-canonical (ERK1/2) pathways which, in turn, lead to the upregulation of downstream genes. Moreover, several studies reported the capability of EMMPRIN as CyPA receptor in activating ERK1/2 signaling pathway [18,26,27].

Thus, we evaluated whether CyPA/EMMPRIN axis may participate in these molecular pathways by means of CyPA administration together with a novel compound as MM284. MM284 selectively block CyPA, is impermeable to cell membranes, and consequently, disables CyPA interaction with EMMPRIN [28].

To this aim, we treated MFS-VSMC with recombinant CyPA with/without MM284 to further investigate the modulation of MFS-related genes and signaling pathways.

Firstly, we confirmed by Western blot that the CyPA treatment significantly increased the EMMPRIN protein expression levels both in HC- and MFS-VSMC. Secondly, we observed that CyPA inhibition restored EMMPRIN levels to those of untreated conditions selectively in MFS-VSMC (Figure 6a). Thirdly, $100 \mathrm{ng} / \mathrm{mL}$ of CyPA treatment upregulates TGFB1 and COL1A1 gene expression levels exclusively in MFS-VSMC, while CyPA inhibitor limited these gene expression levels to the same values of untreated conditions (Figure $6 \mathrm{~b}, \mathrm{c}$ ). These effects were further confirmed also in protein expression levels, since MM284 (250 ng/mL) notably limited CyPA-mediated effects on TGF- $\beta 1$ and collagen protein expression (Figure $6 \mathrm{~d}, \mathrm{e}$ ). At last, the same experimental conditions were adopted to detect the in vitro activation of ERK1/2 signaling. Also, in this case, MFS-VSMC showed an overall upregulation of this pro-fibrotic pathway in comparison with HC-VSMC, the CyPA treatment further enhanced phospho-ERK1/2 expression levels, while MM284 $(250 \mathrm{ng} / \mathrm{mL})$ mitigated CyPA effects in this signaling pathway stimulation (Figure $6 \mathrm{f}$ ).

Taking all these data together, we demonstrated the critical role played by CyPA/EMMPRIN axis in the modulation of MFS-related key pathway activation and gene expression, providing a proof-of-concept that the blockage of this axis may offer a novel therapeutic target to treat TAA in MFS patients. 


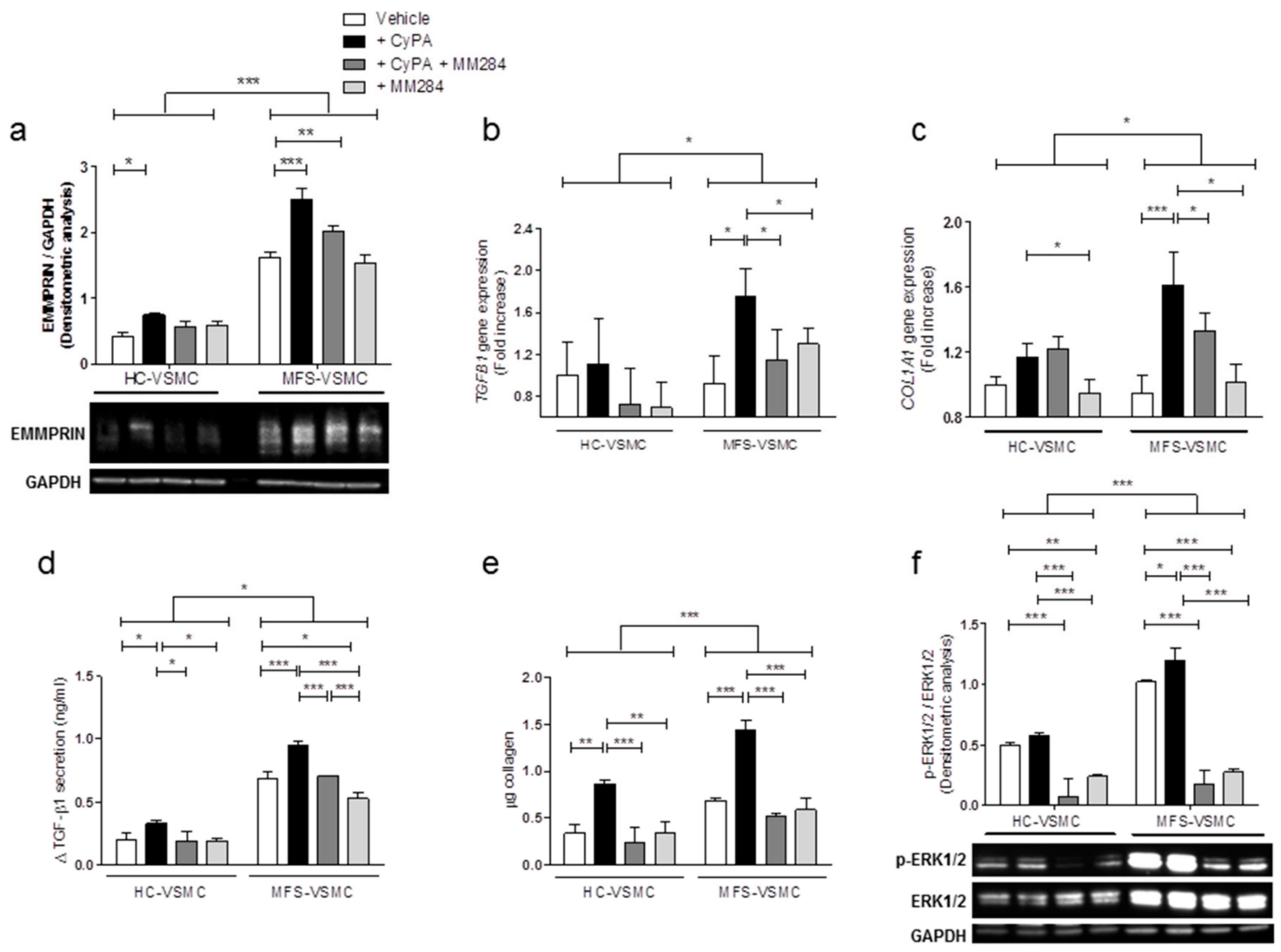

Figure 6. CyPA treatment activates ERK1/2 pathway in MFS-VSMC and promotes pro-fibrotic gene upregulation. (a) EMMPRIN expression levels in HC-VSMC and MFS-VSMC, after 100 ng/mL CyPA, 250ng/mL MM284, and treatment with both molecules. Protein quantification data are shown as mean $\pm \mathrm{SD}, \mathrm{n}=5$. GAPDH has been used as loading control. Two-way ANOVA and Bonferroni's post-test: ${ }^{*} p<0.05,{ }^{* *} p<0.01,{ }^{* * *} p<0.0001$. (b,c) TGFB1 (b) and COL1A1 (c) gene expression in RNA extracts of HC-VSMC and MFS-VSMC, following treatment with $100 \mathrm{ng} / \mathrm{mL} \mathrm{CyPA,} \mathrm{250ng/mL}$ of MM284, and CyPA+MM284. qRT-PCR analyses have been performed three times in triplicate and data are shown as fold change $\pm \mathrm{SD}, \mathrm{n}=5$. Two-way ANOVA and Bonferroni's post-test: ${ }^{*} p<0.05$, *** $p<0.0001$. (d) ELISA assay for TGF- $\beta 1$ in HC-VSMC and MFS-VSMC conditioned medium, after five days of treatment with $100 \mathrm{ng} / \mathrm{mL}$ CyPA, $250 \mathrm{ng} / \mathrm{mL}$ MM284, and CyPA + MM284. Analyses have been performed three times in triplicate and data are shown as mean $\pm \mathrm{SD}, \mathrm{n}=5$. Two-way ANOVA and Bonferroni's post-test: ${ }^{*} p<0.05,{ }^{* * *} p<0.0001$. (e) Quantification of total collagen levels in HC-VSMC and MFS-VSMC after five days of treatment with 100 ng/mL CyPA, 250 ng/mL MM284, and $\mathrm{CyPA}+\mathrm{MM} 284$. Experiments have been performed three times in triplicate and data are shown as mean $\pm \mathrm{SD}, \mathrm{n}=5$. Two-way ANOVA and Bonferroni's post-test: ${ }^{*} p<0.05,{ }^{* *} p<0.01,{ }^{* * *} p<0.001$. (f) Western Blot for active phospho-ERK1/2 and total ERK1/2 proteins in protein extracts of HC-VSMC and MFS-VSMC after 100 ng/mL CyPA, 250ng/mL MM284, and CyPA+MM284 treatment. Protein quantification data are shown as mean $\pm \mathrm{SD}, \mathrm{n}=5$. Total ERK1/2 has been used as loading control. Two-way ANOVA and Bonferroni's post-test: ${ }^{*} p<0.05,{ }^{* *} p<0.01,{ }^{* * *} p<0.0001$.

\section{Discussion}

This study addressed, for the first time, a strong involvement of CyPA/EMMPRIN axis in the modulation of detrimental processes, strictly connected to fibrosis, leading to TAA development of patients with MFS disease. Specifically, we discovered that the in vitro treatment of VSMC isolated by MFS patients' aortas with exogenously administered CyPA leads to: (i) the overexpression and activation of the CyPA receptor EMMPRIN, (ii) the over-activation of ERK1/2 signaling pathway, and (iii) the subsequent upregulation of typical pro-fibrotic mediators, such as TGF- $\beta 1$ and collagen I. 
Altogether, these data suggest a deep involvement of CyPA/EMMPRIN axis is crucial and detrimental processes involved in MFS-associated TAA.

Primum movens of our hypothesis started from the analysis of massive collagen deposition in the dilated zone of MFS patients' thoracic aorta after surgery. This initial observation suggested a larger progression of the fibrotic process in these subjects and was supported by previous insights on the pro-fibrotic histological changes of the aneurysmal aorta [29]. Over time, several studies discovered that TGF- $\beta$ and AngII actively participate as causative players in the aortic aneurysmal development [30]. More specifically, fibrillin- 1 alterations actively contribute to the TGF- $\beta$ massive release from its latent form stored in thoracic aortic ECM [31]. Our histological data was further confirmed at a molecular level by the overexpression of disease-related genes and the activation of specific pro-fibrotic signaling pathways.

On the basis of this strong rationale offered by these remarks, we wanted to in-depth investigate the involvement of CyPA/EMMPRIN axis in the MFS aortic pathological context. In fact, an important involvement of both CyPA and EMMPRIN in several cardiovascular diseases and also in tissue remodeling has been already reported by literature [9]. More specifically, CyPA was reported to be critically involved in vascular remodeling [32], cardiac hypertrophy [33], as well as in AAA formation [17], where its release by VSMC negatively contributes in disease development. Concerning EMMPRIN, this molecule was object of study in atherosclerosis [34,35], cardiac fibrosis in a pressure overload murine model [36], scar tissue formation after myocardial infarction [37], inflammatory cardiomyopathy [10], and tissue remodeling [12,13], revealing its major activity in MMP and pro-fibrotic signaling activation. Importantly, among all these cardiovascular pathologies, Chen et al. reported an AngII-dependent increase in EMMPRIN expression levels on VSMC, in non-syndromic form of both TAA and AAA [11]. Interestingly, our results on EMMPRIN gene and protein expression levels confirmed an upregulation in MFS aortic tissues, particularly in the dilated zone of aortic aneurysm, which is at the same time the most fibrotic aortic region in these patients. Corroborating these results, we recently observed diminished plasmatic levels of the soluble form of EMMPRIN in MFS patients, confirming the detrimental role played by EMMPRIN when its presence is localized on VSMC of aortic tissue [38]. On the other hand, the CyPA gene and protein levels did not reveal any significant difference in comparison with healthy controls. These counter-intuitive results might be explained by a different role/fate of CyPA in this pathological scenario. Different studies on intracellular CyPA reported a cytosolic localization of this molecule, but it is also well known that in the AAA context CyPA is secreted by VSMC after AngII stimulation [17]. Furthermore, this archetypal cell type of aortic wall is the foremost cellular element involved in MFS pathogenesis [39]. Noteworthy, unlike other adult cells, VSMC maintain notable plasticity [40], together with a peculiar ability to alternatively have a contractile or a synthetic phenotype. The latter renders VSMC more proliferative and also source of production of detrimental pro-fibrotic factors, as shown in Figure 7 [40-42]. This feature may be responsible for a larger area of MFS-VSMC in comparison with HC-VSMC that we observed in our experiments. A previous observation of AngII effects on VSMC together with the higher levels of AT1R in MFS samples led us to test this treatment also in our in vitro model of MFS-derived VSMC, in which we appreciated a massive secretion of CyPA specifically in conditioned medium of VSMC obtained from MFS thoracic aortas. This result met an astonishing analogy with the data concerning CyPA concentration in plasma samples of our MFS patients, confirming that the in vitro suggestions may have a pathophysiological counterpart in vivo.

Consistently, we found a strong upregulation of EMMPRIN gene and protein levels after CyPA treatment, corroborating the promoting activity of CyPA on the expression levels of its receptor in our patient-derived VSMC model. Previous studies reported that CyPA/EMMPRIN axis positively modulates ERK1/2 signaling pathway [43-46]. Our results confirm both the upregulation of ERK1/2 pathway after CyPA treatment and the subsequent overexpression of downstream pro-fibrotic mediators. Altogether, these results led us to hypothesize the involvement of the CyPA/EMMPRIN axis activation in the tissue remodeling of thoracic aorta in MFS patients. While the TGF- $\beta$ secretion and collagen 
deposition are important hallmarks of fibrosis, MMPs play a crucial role in tissue remodeling. In fact, a determinant event in the pro-fibrotic ECM remodeling is represented by the activation of MMPs; more specifically, Segura et al. showed a strong overexpression of MMP-2 and MMP-9 in the aortic tunica media of MFS patients [47]. Moreover from further literature emerges the fundamental role of EMMPRIN in MMP-2 and -9 activation in different tissues and pathologies [12,13], including AAA [11]. The results of our work suggested the participation of EMMPRIN in MFS disease, not only as a receptor activating the ERK1/2 pro-fibrotic signaling pathway but also as an inducer of the MMP-2 activation.

In addition, it has been reported that the CyPA binding to EMMPRIN is necessary to enable it as an MMP inducer and the results collected demonstrate the proper functioning of this axis [48]. It is also worth it to point out that the fibrotic process can be considered as a double edge sword in cardiovascular pathophysiology, being, at the same time, an adaptive and protective mechanism, but also an irreversible remodeling process impairing organ function and architecture [49]. Therefore, fibrosis into the aortic wall should not be considered a pathological event, unless it becomes detrimental when passing an unknown threshold, who's definition is not within the scope of this work. Nonetheless, unraveling a new pro-fibrotic central axis in MFS TAA may add new information for future investigations.

These results open a new perspective for TAA pharmacological research, which avoids direct TGF- $\beta 1$ targeting. In the last years, in fact, published studies have generated conflicting results when targeting TGF- $\beta 1$ itself and/or its downstream pathways for TAA treatment [7,8,50-52]. Of note, our experiments, taking advantage of in vitro blockade of the CyPA/EMMPRIN axis by MM284, further highlighted a strong involvement of this axis in TAA of MFS by reverting pro-fibrotic pathways including the secretion of the active form of TGF- $\beta 1$. These results hint at a possible exploitation of cyclosporine analogs in counteracting TAA development in MFS. In this regard, additional in vivo experiments are needed to confirm this hypothesis. Several studies already provided evidence on MM284 beneficial role in cardiovascular conditions, such as myocarditis [53] and thrombi formation [54].

\section{Conclusions}

In conclusion, our results, summarized in Figure 7, demonstrate that AngII-related CyPA secretion can biochemically and functionally activate EMMPRIN in the dilated portion of MFS patients' aorta by promoting the production of pro-fibrotic mediators and by simultaneously activating MMP. Since an etiological treatment for TAA is still lacking [39], this work has the potential to pave the way to new druggable pathways to be exploited for pharmacological TAA treatment development.

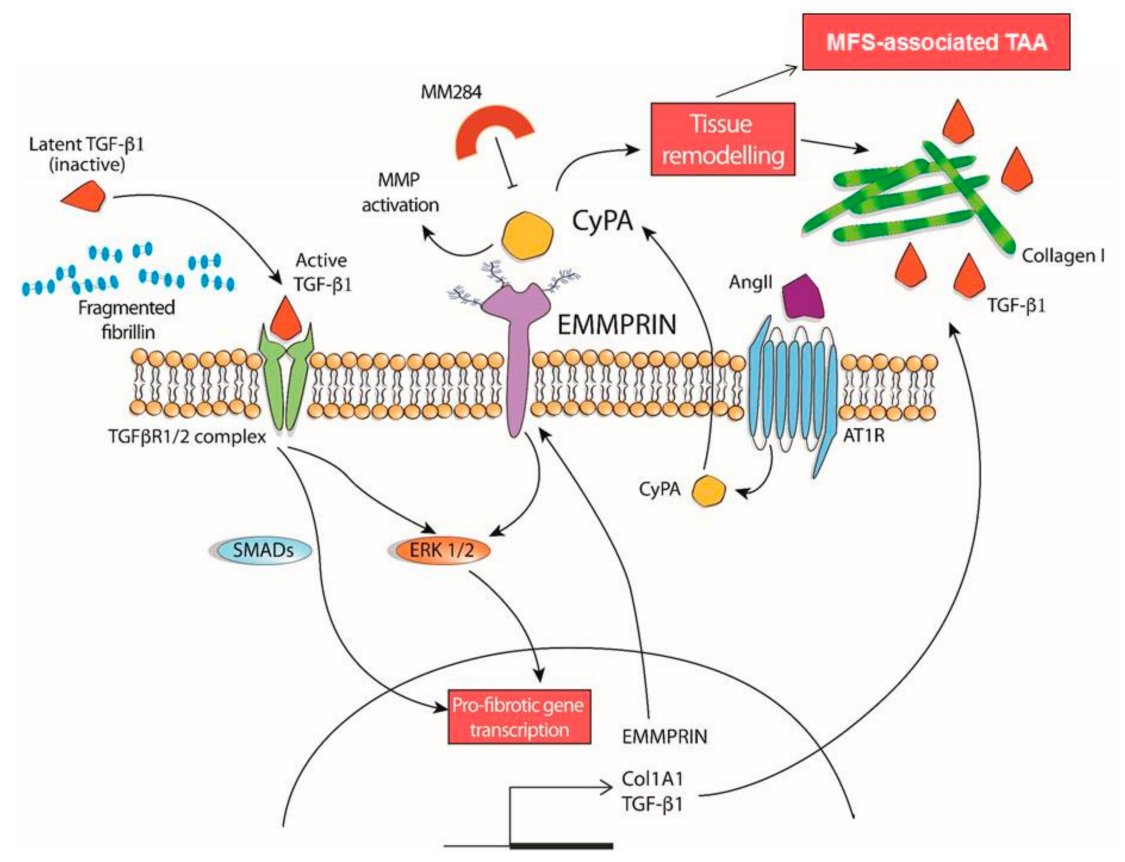


Figure 7. Proposed mechanism of CyPA/EMMPRIN axis involvement in TAA associated with MFS.The ECM of thoracic aorta in MFS patients is characterized by fragmented fibrillin-1, which determines TGF- $\beta 1$ release. Active TGF- $\beta 1$ binds its receptor and stimulates both the SMAD2/3 (canonical) and ERK1/2 (non-canonical) pathways, leading to the transcription and translation of pro-fibrotic factors, such as collagen and TGF- $\beta 1$ itself. AngII stimulus leads to the secretion of CyPA, which binds its receptor EMMPRIN. The CyPA/EMMPRIN axis triggers (i) ERK1/2 pathway (non-canonical signaling, shared with TGF- $\beta 1$ ), (ii) EMMPRIN, which, in turn, induces MMP activation, and (iii) the expression of typical downstream ERK1/2 pro-fibrotic mediators, involved in tissue remodeling of MFS-associated TAA. The inhibitor MM284 reverses all the deleterious effects of CyPA in MFS-VSMC.

Supplementary Materials: The following are available online at http://www.mdpi.com/2073-4409/9/1/154/s1, Figure S1: Cells isolated from aortic tunica media are VSMC and express CyPA and EMMPRIN; http://www. mdpi.com/2073-4409/9/1/154/s2, Figure S2: CyPA is localized into the cytoplasm of VSMC; http://www.mdpi. com/2073-4409/9/1/154/s3, Figure S3: CyPA does not alter cell proliferation and does not induce apoptosis in MFS-VSMC; http://www.mdpi.com/2073-4409/9/1/154/s4, Figure S4: CyPA/EMMPRIN axis inhibition decreases MMP expression and activity in MFS-VSMC.

Author Contributions: Conceptualization: G.L.P., G.P., and P.N.; funding acquisition: G.P. and P.N.; investigation: G.L.P., E.R., M.C., M.B., M.P., D.M., G.E., G.F., and M.M.; project administration: G.P.; resources: A.P., R.G., C.A., F.A., E.C., A.P., and G.P.; supervision: G.P. and P.N.; data analysis, result inference: G.L.P. and P.N.; writing-original draft: G.L.P. and P.N.; reviewing and editing draft: G.L.P., E.R., M.C., M.B., M.P., D.M., A.P., R.G., C.A., G.E., G.F., M.M., F.A., E.C., A.P., G.P., and P.N. All authors have read and agreed to the published version of the manuscript.

Funding: This work was supported by the National Ministry of Health funds (Ricerca Corrente RC2015-2018).

Acknowledgments: The authors declare no competing financial interests. We thank Barbara Micheli, Anna Guarino and Gianluca Polvani for their valuable contribution and suggestions for the manuscript elaboration.

Conflicts of Interest: The authors declare no conflict of interest.

\section{References}

1. Judge, D.P.; Dietz, H.C. Marfan's syndrome. Lancet 2005, 366, 1965-1976. [CrossRef]

2. Dietz, H.C.; Cutting, G.R.; Pyeritz, R.E.; Maslen, C.L.; Sakai, L.Y.; Corson, G.M.; Puffenberger, E.G.; Hamosh, A.; Nanthakumar, E.J.; Curristin, S.M.; et al. Marfan syndrome caused by a recurrent de novo missense mutation in the fibrillin gene. Nature 1991, 352, 337-339. [CrossRef]

3. Davis, M.R.; Summers, K.M. Structure and function of the mammalian fibrillin gene family: Implications for human connective tissue diseases. Mol. Genet. Metab. 2012, 107, 635-647. [CrossRef]

4. Schrijver, I.; Liu, W.; Brenn, T.; Furthmayr, H.; Francke, U. Cysteine substitutions in epidermal growth factor-like domains of fibrillin-1: Distinct effects on biochemical and clinical phenotypes. Am. J. Hum. Genet. 1999, 65, 1007-1020. [CrossRef]

5. Ammash, N.M.; Sundt, T.M.; Connolly, H.M. Marfan syndrome-diagnosis and management. Curr. Probl. Cardiol. 2008, 33, 7-39. [CrossRef]

6. Rurali, E.; Perrucci, G.L.; Pilato, C.A.; Pini, A.; Gaetano, R.; Nigro, P.; Pompilio, G. Precise Therapy for Thoracic Aortic Aneurysm in Marfan Syndrome: A Puzzle Nearing Its Solution. Prog. Cardiovasc. Dis. 2018, 61, 328-335. [CrossRef]

7. Habashi, J.P.; Judge, D.P.; Holm, T.M.; Cohn, R.D.; Loeys, B.L.; Cooper, T.K.; Myers, L.; Klein, E.C.; Liu, G.; Calvi, C.; et al. Losartan, an AT1 antagonist, prevents aortic aneurysm in a mouse model of Marfan syndrome. Science 2006, 312, 117-121. [CrossRef]

8. Holm, T.M.; Habashi, J.P.; Doyle, J.J.; Bedja, D.; Chen, Y.; van Erp, C.; Lindsay, M.E.; Kim, D.; Schoenhoff, F.; Cohn, R.D.; et al. Noncanonical TGFbeta signaling contributes to aortic aneurysm progression in Marfan syndrome mice. Science 2011, 332, 358-361. [CrossRef] [PubMed]

9. Seizer, P.; Gawaz, M.; May, A.E. Cyclophilin A and EMMPRIN (CD147) in cardiovascular diseases. Cardiovasc. Res. 2014, 102, 17-23. [CrossRef] [PubMed]

10. Seizer, P.; Geisler, T.; Bigalke, B.; Schneider, M.; Klingel, K.; Kandolf, R.; Stellos, K.; Schreieck, J.; Gawaz, M.; May, A.E. EMMPRIN and its ligand cyclophilin A as novel diagnostic markers in inflammatory cardiomyopathy. Int. J. Cardiol. 2013, 163, 299-304. [CrossRef] [PubMed] 
11. Chen, X.F.; Wang, J.A.; Hou, J.; Gui, C.; Tang, L.J.; Chen, X.Q.; Xie, X.J.; Jiang, J.J.; Cai, J.F.; Chen, H.S.; et al. Extracellular matrix metalloproteinase inducer (EMMPRIN) is present in smooth muscle cells of human aneurysmal aorta and is induced by angiotensin II in vitro. Clin. Sci. 2009, 116, 819-826. [CrossRef] [PubMed]

12. Huet, E.; Gabison, E.E.; Mourah, S.; Menashi, S. Role of emmprin/CD147 in tissue remodeling. Connect. Tissue Res. 2008, 49, 175-179. [CrossRef] [PubMed]

13. Huet, E.; Vallee, B.; Szul, D.; Verrecchia, F.; Mourah, S.; Jester, J.V.; Hoang-Xuan, T.; Menashi, S.; Gabison, E.E. Extracellular matrix metalloproteinase inducer/CD147 promotes myofibroblast differentiation by inducing alpha-smooth muscle actin expression and collagen gel contraction: Implications in tissue remodeling. FASEB J. 2008, 22, 1144-1154. [CrossRef] [PubMed]

14. Von Ungern-Sternberg, S.N.I.; Zernecke, A.; Seizer, P. Extracellular Matrix Metalloproteinase Inducer EMMPRIN (CD147) in Cardiovascular Disease. Int. J. Mol. Sci. 2018, 19, E507. [CrossRef]

15. Nigro, P.; Pompilio, G.; Capogrossi, M.C. Cyclophilin A: A key player for human disease. Cell Death Dis. 2013, 4, e888. [CrossRef]

16. Perrucci, G.L.; Gowran, A.; Zanobini, M.; Capogrossi, M.C.; Pompilio, G.; Nigro, P. Peptidyl-prolyl isomerases: A full cast of critical actors in cardiovascular diseases. Cardiovasc. Res. 2015, 106, 353-364. [CrossRef]

17. Satoh, K.; Nigro, P.; Matoba, T.; O’Dell, M.R.; Cui, Z.; Shi, X.; Mohan, A.; Yan, C.; Abe, J.; Illig, K.A.; et al. Cyclophilin A enhances vascular oxidative stress and the development of angiotensin II-induced aortic aneurysms. Nat. Med. 2009, 15, 649-656. [CrossRef]

18. Kim, J.Y.; Kim, H.; Suk, K.; Lee, W.H. Activation of CD147 with cyclophilin a induces the expression of IFITM1 through ERK and PI3K in THP-1 cells. Mediat. Inflamm. 2010, 2010, 821940. [CrossRef]

19. Jin, Z.G.; Melaragno, M.G.; Liao, D.F.; Yan, C.; Haendeler, J.; Suh, Y.A.; Lambeth, J.D.; Berk, B.C. Cyclophilin $\mathrm{A}$ is a secreted growth factor induced by oxidative stress. Circ. Res. 2000, 87, 789-796. [CrossRef]

20. Suzuki, J.; Jin, Z.G.; Meoli, D.F.; Matoba, T.; Berk, B.C. Cyclophilin A is secreted by a vesicular pathway in vascular smooth muscle cells. Circ. Res. 2006, 98, 811-817. [CrossRef]

21. Nataatmadja, M.; West, J.; Prabowo, S.; West, M. Angiotensin II Receptor Antagonism Reduces Transforming Growth Factor Beta and Smad Signaling in Thoracic Aortic Aneurysm. Ochsner J. 2013, 13, 42-48. [PubMed]

22. Xue, C.; Sowden, M.; Berk, B.C. Extracellular Cyclophilin, A.; Especially Acetylated, Causes Pulmonary Hypertension by Stimulating Endothelial Apoptosis, Redox Stress, and Inflammation. Arterioscler. Thromb. Vasc. Biol. 2017, 37, 1138-1146. [CrossRef] [PubMed]

23. Ramachandran, S.; Venugopal, A.; Kutty, V.R.; Vinitha, A.; Divya, G.; Chitrasree, V.; Kartha, C.C. Plasma level of cyclophilin $\mathrm{A}$ is increased in patients with type 2 diabetes mellitus and suggests presence of vascular disease. Cardiovasc. Diabetol. 2014, 13, 38. [CrossRef] [PubMed]

24. Yan, J.; Zang, X.; Chen, R.; Yuan, W.; Gong, J.; Wang, C.; Li, Y. The clinical implications of increased cyclophilin A levels in patients with acute coronary syndromes. Clin. Chim. Acta 2012, 413, 691-695. [CrossRef]

25. Hattori, F. Extracellular cyclophilin A as a humoral factor modulating cardiovascular inflammatory responses. J. Mol. Cell Cardiol. 2012, 53, 1-2. [CrossRef]

26. Li, H.Y.; Ju, D.; Zhang, D.W.; Li, H.; Kong, L.M.; Guo, Y.; Li, C.; Wang, X.L.; Chen, Z.N.; Bian, H. Activation of TGF-beta1-CD147 positive feedback loop in hepatic stellate cells promotes liver fibrosis. Sci. Rep. 2015, 5, 16552. [CrossRef]

27. Yang, H.; Chen, J.; Yang, J.; Qiao, S.; Zhao, S.; Yu, L. Cyclophilin A is upregulated in small cell lung cancer and activates ERK1/2 signal. Biochem. Biophys Res. Commun. 2007, 361,763-767. [CrossRef]

28. Malesevic, M.; Gutknecht, D.; Prell, E.; Klein, C.; Schumann, M.; Nowak, R.A.; Simon, J.C.; Schiene-Fischer, C.; Saalbach, A. Anti-inflammatory effects of extracellular cyclosporins are exclusively mediated by CD147. J. Med. Chem. 2013, 56, 7302-7311. [CrossRef]

29. Peterss, S.; Mansour, A.M.; Ross, J.A.; Vaitkeviciute, I.; Charilaou, P.; Dumfarth, J.; Fang, H.; Ziganshin, B.A.; Rizzo, J.A.; Adeniran, A.J.; et al. Changing Pathology of the Thoracic Aorta From Acute to Chronic Dissection: Literature Review and Insights. J. Am. Coll. Cardiol. 2016, 68, 1054-1065. [CrossRef]

30. Chen, X.; Lu, H.; Rateri, D.L.; Cassis, L.A.; Daugherty, A. Conundrum of angiotensin II and TGF-beta interactions in aortic aneurysms. Curr. Opin. Pharm. 2013, 13, 180-185. [CrossRef]

31. Chaudhry, S.S.; Cain, S.A.; Morgan, A.; Dallas, S.L.; Shuttleworth, C.A.; Kielty, C.M. Fibrillin-1 regulates the bioavailability of TGFbeta1. J. Cell Biol. 2007, 176, 355-367. [CrossRef] [PubMed] 
32. Satoh, K.; Matoba, T.; Suzuki, J.; O’Dell, M.R.; Nigro, P.; Cui, Z.; Mohan, A.; Pan, S.; Li, L.; Jin, Z.G.; et al. Cyclophilin A mediates vascular remodeling by promoting inflammation and vascular smooth muscle cell proliferation. Circulation 2008, 117, 3088-3098. [CrossRef] [PubMed]

33. Satoh, K.; Nigro, P.; Zeidan, A.; Soe, N.N.; Jaffre, F.; Oikawa, M.; O’Dell, M.R.; Cui, Z.; Menon, P.; Lu, Y.; et al. Cyclophilin A Promotes Cardiac Hypertrophy in Apolipoprotein E-Deficient Mice. Arterioscler. Thromb. Vasc. Biol. 2011, 31, 1116-1123. [CrossRef] [PubMed]

34. Yoon, Y.W.; Kwon, H.M.; Hwang, K.C.; Choi, E.Y.; Hong, B.K.; Kim, D.; Kim, H.S.; Cho, S.H.; Song, K.S.; Sangiorgi, G. Upstream regulation of matrix metalloproteinase by EMMPRIN; extracellular matrix metalloproteinase inducer in advanced atherosclerotic plaque. Atherosclerosis 2005, 180, 37-44. [CrossRef] [PubMed]

35. Seizer, P.; Schonberger, T.; Schott, M.; Lang, M.R.; Langer, H.F.; Bigalke, B.; Kramer, B.F.; Borst, O.; Daub, K.; Heidenreich, O.; et al. EMMPRIN and its ligand cyclophilin A regulate MT1-MMP, MMP-9 and M-CSF during foam cell formation. Atherosclerosis 2010, 209, 51-57. [CrossRef] [PubMed]

36. Suzuki, K.; Satoh, K.; Ikeda, S.; Sunamura, S.; Otsuki, T.; Satoh, T.; Kikuchi, N.; Omura, J.; Kurosawa, R.; Nogi, M.; et al. Basigin Promotes Cardiac Fibrosis and Failure in Response to Chronic Pressure Overload in Mice. Arterioscler. Thromb. Vasc. Biol. 2016, 36, 636-646. [CrossRef]

37. Seizer, P.; Ochmann, C.; Schonberger, T.; Zach, S.; Rose, M.; Borst, O.; Klingel, K.; Kandolf, R.; MacDonald, H.R.; Nowak, R.A.; et al. Disrupting the EMMPRIN (CD147)-cyclophilin A interaction reduces infarct size and preserves systolic function after myocardial ischemia and reperfusion. Arterioscler. Thromb. Vasc. Biol. 2011, 31, 1377-1386. [CrossRef]

38. Rurali, E.; Perrucci, G.L.; Gaetano, R.; Pini, A.; Moschetta, D.; Gentilini, D.; Nigro, P.; Pompilio, G. Soluble EMMPRIN levels discriminate aortic ectasia in Marfan syndrome patients. Theranostics 2019, 9, 2224-2234. [CrossRef]

39. Perrucci, G.L.; Rurali, E.; Gowran, A.; Pini, A.; Antona, C.; Chiesa, R.; Pompilio, G.; Nigro, P. Vascular smooth muscle cells in Marfan syndrome aneurysm: The broken bricks in the aortic wall. Cell Mol. Life Sci. 2017, 74, 267-277. [CrossRef]

40. Nguyen, A.T.; Gomez, D.; Bell, R.D.; Campbell, J.H.; Clowes, A.W.; Gabbiani, G.; Giachelli, C.M.; Parmacek, M.S.; Raines, E.W.; Rusch, N.J.; et al. Smooth muscle cell plasticity: Fact or fiction? Circ. Res. 2013, 112, 17-22. [CrossRef]

41. Li, Z.; Zhao, X.; Bai, S.; Wang, Z.; Chen, L.; Wei, Y.; Huang, C. Proteomics identification of cyclophilin a as a potential prognostic factor and therapeutic target in endometrial carcinoma. Mol. Cell Proteom. 2008, 7, 1810-1823. [CrossRef]

42. Bunton, T.E.; Biery, N.J.; Myers, L.; Gayraud, B.; Ramirez, F.; Dietz, H.C. Phenotypic alteration of vascular smooth muscle cells precedes elastolysis in a mouse model of Marfan syndrome. Circ. Res. 2001, 88, 37-43. [CrossRef] [PubMed]

43. Yurchenko, V.; Zybarth, G.; O'Connor, M.; Dai, W.W.; Franchin, G.; Hao, T.; Guo, H.; Hung, H.C.; Toole, B.; Gallay, P.; et al. Active site residues of cyclophilin A are crucial for its signaling activity via CD147. J. Biol. Chem. 2002, 277, 22959-22965. [CrossRef] [PubMed]

44. Venkatesan, B.; Valente, A.J.; Reddy, V.S.; Siwik, D.A.; Chandrasekar, B. Resveratrol blocks interleukin-18EMMPRIN cross-regulation and smooth muscle cell migration. Am. J. Physiol. Heart Circ. Physiol. 2009, 297, H874-H886. [CrossRef] [PubMed]

45. Yuan, W.; Ge, H.; He, B. Pro-inflammatory activities induced by CyPA-EMMPRIN interaction in monocytes. Atherosclerosis 2010, 213, 415-421. [CrossRef]

46. Qu, X.; Wang, C.; Zhang, J.; Qie, G.; Zhou, J. The roles of CD147 and/or cyclophilin A in kidney diseases. Mediat. Inflamm. 2014, 2014, 728673. [CrossRef] [PubMed]

47. Segura, A.M.; Luna, R.E.; Horiba, K.; Stetler-Stevenson, W.G.; McAllister, H.A., Jr.; Willerson, J.T.; Ferrans, V.J. Immunohistochemistry of matrix metalloproteinases and their inhibitors in thoracic aortic aneurysms and aortic valves of patients with Marfan's syndrome. Circulation 1998, 98, II331-II337. [PubMed]

48. Wang, C.H.; Dai, J.Y.; Wang, L.; Jia, J.F.; Zheng, Z.H.; Ding, J.; Chen, Z.N.; Zhu, P. Expression of CD147 (EMMPRIN) on neutrophils in rheumatoid arthritis enhances chemotaxis, matrix metalloproteinase production and invasiveness of synoviocytes. J. Cell Mol. Med. 2011, 15, 850-860. [CrossRef]

49. Perrucci, G.L.; Rurali, E.; Pompilio, G. Cardiac fibrosis in regenerative medicine: Destroy to rebuild. J. Thorac. Dis. 2018, 10, S2376-S2389. [CrossRef] 
50. Cook, J.R.; Clayton, N.P.; Carta, L.; Galatioto, J.; Chiu, E.; Smaldone, S.; Nelson, C.A.; Cheng, S.H.; Wentworth, B.M.; Ramirez, F. Dimorphic effects of transforming growth factor-beta signaling during aortic aneurysm progression in mice suggest a combinatorial therapy for Marfan syndrome. Arterioscler. Thromb. Vasc. Biol. 2015, 35, 911-917. [CrossRef]

51. Li, W.; Li, Q.; Jiao, Y.; Qin, L.; Ali, R.; Zhou, J.; Ferruzzi, J.; Kim, R.W.; Geirsson, A.; Dietz, H.C.; et al. Tgfbr2 disruption in postnatal smooth muscle impairs aortic wall homeostasis. J. Clin. Investig. 2014, 124, 755-767. [CrossRef] [PubMed]

52. Milewicz, D.M.; Ramirez, F. Therapies for Thoracic Aortic Aneurysms and Acute Aortic Dissections. Arterioscler. Thromb. Vasc. Biol. 2019, 39, 126-136. [CrossRef] [PubMed]

53. Heinzmann, D.; Bangert, A.; Muller, A.M.; von Ungern-Sternberg, S.N.; Emschermann, F.; Schonberger, T.; Chatterjee, M.; Mack, A.F.; Klingel, K.; Kandolf, R.; et al. The Novel Extracellular Cyclophilin A (CyPA) - Inhibitor MM284 Reduces Myocardial Inflammation and Remodeling in a Mouse Model of Troponin I -Induced Myocarditis. PLoS ONE 2015, 10, e0124606. [CrossRef] [PubMed]

54. Seizer, P.; Ungern-Sternberg, S.N.; Schonberger, T.; Borst, O.; Munzer, P.; Schmidt, E.M.; Mack, A.F.; Heinzmann, D.; Chatterjee, M.; Langer, H.; et al. Extracellular cyclophilin A activates platelets via EMMPRIN (CD147) and PI3K/Akt signaling, which promotes platelet adhesion and thrombus formation in vitro and in vivo. Arterioscler. Thromb. Vasc. Biol. 2015, 35, 655-663. [CrossRef] [PubMed]

(C) 2020 by the authors. Licensee MDPI, Basel, Switzerland. This article is an open access article distributed under the terms and conditions of the Creative Commons Attribution (CC BY) license (http://creativecommons.org/licenses/by/4.0/). 